\title{
Driver's ECG Signal Detection and Transmission by Impulse-Radio-Based Human Body Communication Technology
}

\author{
Jianqing Wang \\ Graduate School of \\ Engineering \\ Nagoya Institute of Technology \\ Nagoya 466-8555, Japan \\ wang@nitech.ac.jp
}

\author{
Taku Kato \\ Graduate School of \\ Engineering \\ Nagoya 466-8555, Japan \\ 25417509@stn.nitech.ac.jp
}

Nagoya Institute of Technology Nagoya Institute of Technology

\author{
Daisuke Anzai \\ Graduate School of \\ Engineering \\ Nagoya 466-8555, Japan \\ anzai@nitech.ac.jp
}

\begin{abstract}
In this study, we developed a wearable electrocardiogram (ECG) sensor with human body communication (HBC) technology for vital data transmission in a car. The ECG signals were modulated with wideband pulse signals between 10 and $60 \mathrm{MHz}$ based on an impulse radio (IR) scheme, which provides a data rate as high as $1.25 \mathrm{Mbps}$. To apply the HBC-based wearable ECG in a moving car, we employed a moving average algorithm to reduce the noises produced in the received ECG signals due to the car's movement. As a result, we confirmed a real-time ECG transmission of driver in the moving car with a reasonable accuracy.
\end{abstract}

\section{Categories and Subject Descriptors}

C.2.1 [Computer-Communication Networks]: Network Architecture and Design-Wireless Communication

\section{General Terms}

Experimentation

\section{Keywords}

Human body communication, impulse radio transceiver, electrocardiogram

\section{INTRODUCTION}

There is a wide-scale demand for health-state monitoring in various environments [1][2]. One of them is the monitoring of driver's health-state for safe driving. In this scenario, some vital sign sensors are placed on the driver's body to collect signals such as ECG, blood presser and pulse rate. The ECG sensor may be embedded in the driver's seat belt, and the access point may be embedded in the steering wheel so that the driver unconsciously wears the sensors and send the data to an access point by a wireless or HBC technology [3]-[5]. The car's control unit can then analyze the driver's

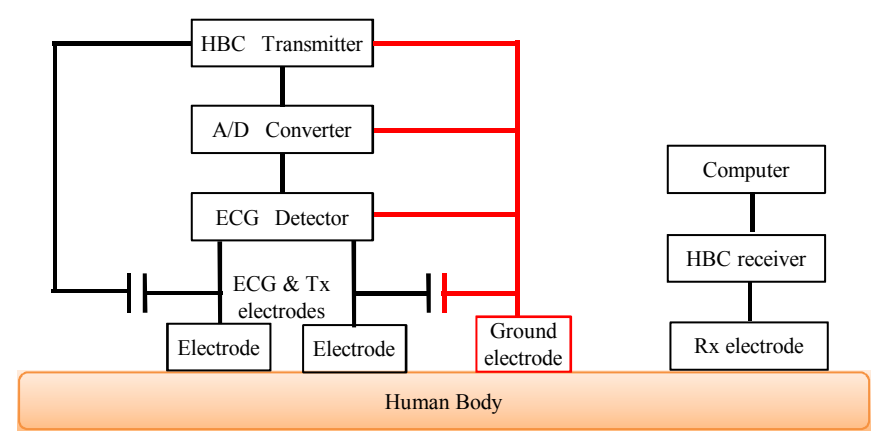

Figure 1: Structure of wearable ECG.

health-state data collected in the access point and generate warning signs or take automatic control of car, if necessary.

In this study, we develop a wearable ECG sensor with HBC technology for real time monitoring. Instead of a sinusoid signal, digitized ECG signals are modulated with wide band pulses between 10 and $60 \mathrm{MHz}$, which is known as impulse radio (IR) scheme [6]. Such a wide band transmission can provide advantages such as high data rate and antiinterference feature. Its low power consumption also contributes to a long time use. After the development of the HBC-based wearable ECG, we apply it in a moving car for real time transmission of driver's ECG signals. We introduce a moving average algorithm to reduce the noises produced in the received ECG signals due to the car's movement, and demonstrate the usefulness of the developed wearable ECG in the moving car.

\section{HBC-BASED WEARABLE ECG}

Fig. 1 shows the structure of our developed HBC-based wearable ECG. The ECG sensor is composited of two $3 \mathrm{~cm}$ $\times 3 \mathrm{~cm}$ square electrodes. The two electrodes are attached to the chest for ECG signal sensing. A ground electrode is further attached to the human body as a reference. The ECG signals acquired by the two sensing electrodes are filtered and differentially amplified in the ECG detector, and then converted to digital signals by an analog-to-digital (AD) converter. The $A D$-converted ECG signals are transmitted by the $\mathrm{HBC}$ transmitter to a $\mathrm{HBC}$ receiver through the hu- 
Table 1: IR transceiver specifications

\begin{tabular}{l|c}
\hline Frequency band & $10-60 \mathrm{MHz}$ \\
\hline Modulation & IR-PPM \\
\hline Data Rate & $1.25 \mathrm{Mbps}$ \\
\hline Maximum output & $-15 \mathrm{dBm}$ \\
\hline Demodulation & Energy detection \\
\hline
\end{tabular}

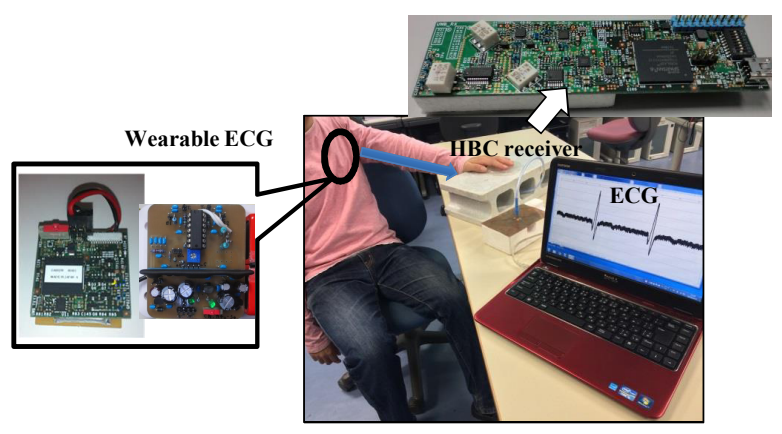

Figure 2: View of verification experiment.

man body. The two transmit electrodes are common as the two sensing electrodes. In the ECG signal sensing, the two sensing electrodes act both as the signal electrodes. However, in the HBC transmission, one of them acts as signal electrode, and the other acts as the ground electrode. To make the two electrodes be commonly used, we insert two capacitors, as shown in Fig. 1, to make their DC potential be different. Such a structure makes the two electrodes can act for not only signal sensing but also signal transmission. Moreover, the low pass filter (LPF) in the ECG detector is designed yo also avoid the mixture of the HBC pulses into the ECG signals. The ECG signals are transmitted by the two common-use electrodes along the human body to the $\mathrm{HBC}$ receiver. The HBC receiver has a universal serial bus (USB) interface via which the received ECG signals can be sent to a computer for further process or display.

The digitized ECG signals are modulated with wide band pulses based on IR scheme in the transmitter. The transmit pulses are produced by a $5-\mathrm{MHz}$ oscillator. The XOR operation of the pulse sequence at $5 \mathrm{MHz}$ and its delayed version with a delay time of $10 \mathrm{~ns}$ are made to produce a new pulse sequence. The pulse width in the new pulse sequence is around $10 \mathrm{~ns}$ and the pulse number is doubled. Its spectrum shape is formed between 10 and $60 \mathrm{MHz}$ with appropriate filtering. In order to transmit the digitized data bits, we employ multiple pulse positions to represent bits " 1 " and " 0 ", which is actually a pulse position modulation (PPM) scheme at a date rate of $1.25 \mathrm{Mbps}$. The HBC receiver employs an envelope detector for demodulation. The received signal is filtered and amplified, and is then adjusted to an adequate level by an automatic gain controller for demodulation. Table 1 summarizes the basic specifications of the IR-type HBC transceiver.

\section{VERIFICATION EXPERIMENT}

The validity of the developed HBC-based wearable ECG was verified by comparison of the measured results between our wearable ECG and a commercial available Holter ECG
Table 2: Comparison of correlation coefficient of RRI

\begin{tabular}{c|c|c|c}
\hline $\mathrm{A}$ & $\mathrm{B}$ & $\mathrm{C}$ & Average \\
\hline 0.953 & 0.984 & 0.972 & 0.970 \\
\hline
\end{tabular}

Table 3: Comparison of RR50 (Sample/min)

\begin{tabular}{l|c|c|c|c}
\hline & A & B & C & Average \\
\hline Wearable & 18.8 & 12.3 & 7.8 & 13.0 \\
Holter & 17.7 & 11.3 & 8.3 & 12.4 \\
Difference (\%) & 5.8 & 8.7 & 6.5 & 7.0 \\
\hline
\end{tabular}

(Fukuda Denshi Co. Ltd.). We set both our wearable ECG and the Holter ECG on the chest, and acquired the ECG signal simultaneously. Fig. 2 shows the view of ECG acquisition and transmission experiment. This experiment was conducted for three persons ( A, B and C). For the acquired ECG signals from the three persons, we made a heart rate variability analysis, and extracted the inter-beat (RR) interval (RRI) and the number that the RR difference is larger than $50 \mathrm{~ms}$ per minute (RR50). Table 2 and 3 compare the RRI and RR50 between our wearable ECG and the Holter ECG. As can be seen, the correlation coefficient is higher than 0.953 for RRI, and the average difference is only $7.0 \%$ for RR50. Moreover, we also compared the low-frequency $(0.05-0.15 \mathrm{~Hz})$ to high-frequency $(0.15-0.40 \mathrm{~Hz})(\mathrm{LF} / \mathrm{HF})$ ratio for the acquired ECG signals. The LF/HF ratio is usually used as an index of cardiac sympathetic modulation. Table 4 compares the LF/HF ratio between our wearable ECG and the Holter ECG. Again, the average difference is only $4.4 \%$. Such a good correlation and low differences suggest that our wearable ECG has the same performance as commercial Holter ECG.

\section{ECG TRANSMISSION IN CAR}

After the performance validation for our developed wearable ECG, we applied it in a moving car to acquire and transmit the driver's ECG signal to a personal computer in the front of the car. Fig. 3 shows the view of the experiment. The wearable ECG was set on the chest of the driver. When the driver's hand touched the receiver electrodes, his ECG signal was detected and sent to the receiver and then displayed on the PC screen in a real time.

Fig. 4 shows two examples of acquired ECG signals. One is that when the car stopped, and the other is that when the car ran at a speed of about $30 \mathrm{~km} / \mathrm{h}$. As can be seen in the figures, not only the Q-, R-, S-waves but also the P- and Twaves were observed in the ECG signals even when the car was running. The results demonstrate a sufficient feasibility of our wearable ECG for driver's ECG monitoring. However, it was also found that more noise components appeared in the ECG signals when driving. The $\mathrm{P}$-wave and T-wave were especially affected by the noises. By performing a Fourier

Table 4: Comparison of $\mathrm{LF} / \mathrm{HF}$

\begin{tabular}{l|c|c|c|c}
\hline & A & B & C & Average \\
\hline Wearable & 0.76 & 1.86 & 1.51 & 1.38 \\
Holter & 0.73 & 1.78 & 1.44 & 1.32 \\
Difference (\%) & 4.2 & 4.6 & 4.4 & 4.4 \\
\hline
\end{tabular}




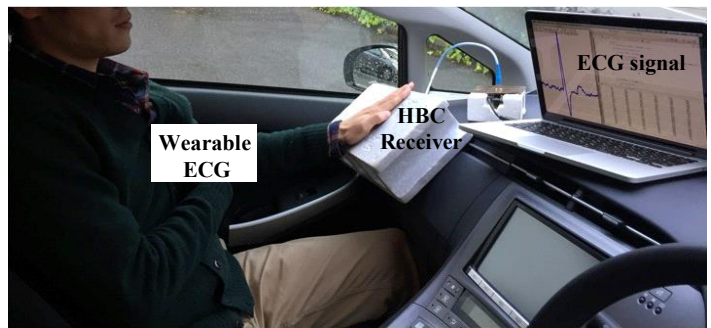

Figure 3: ECG acquisition and transmission in a moving car.
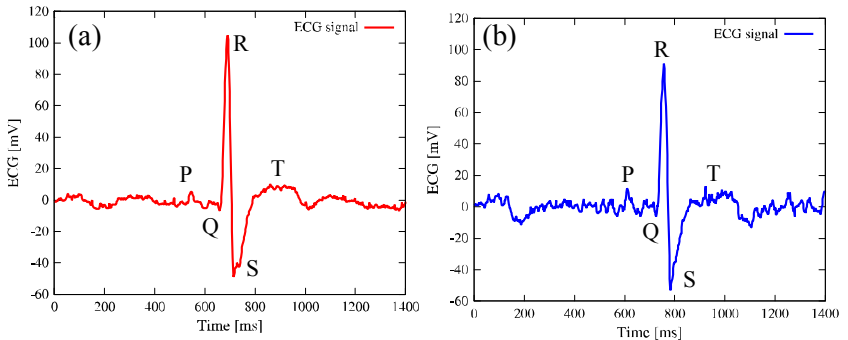

Figure 4: Acquired ECG signals. (a) Stopping, (b) driving.

transform for the noise components in the ECG signals, we found that the noise components were mainly between 30 and $50 \mathrm{~Hz}$. We then introduced a moving average filter in the receiver side. The moving average filter was designed to have 10 taps for removing the frequency components from 30 to $50 \mathrm{~Hz}$. As shown in Fig. 5, the noise components in driving were attenuated about $40 \mathrm{~dB}$ by the moving average filter. Fig. 6 shows the ECG signal after the moving average filter when driving. The ECG signal quality was significantly improved. Such a filter is easily realized in the receiver side, and it almost does not yield any obvious signal delay. In our experiment, we implemented it in PC by software.

\section{CONCLUSION}

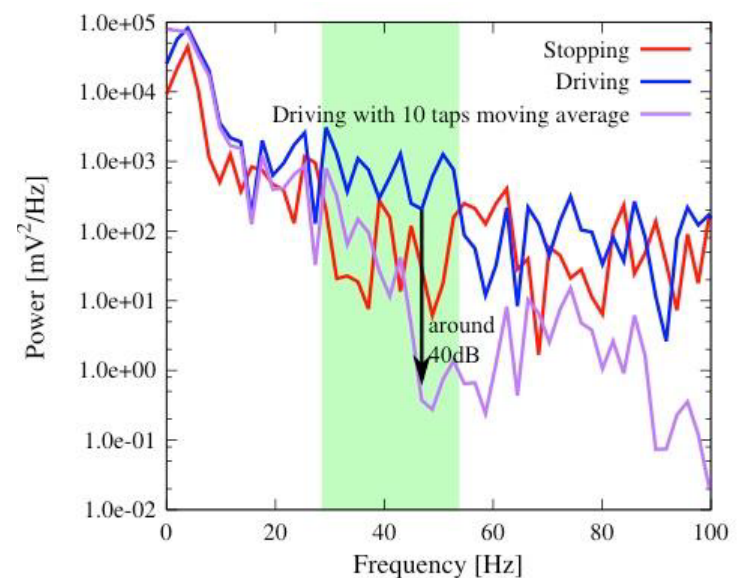

Figure 5: Frequency spectra of acquired ECG signals.

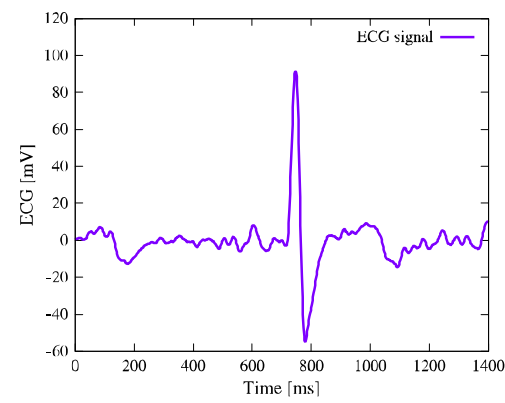

Figure 6: ECG signal processed by moving average filter when driving.

As a promising application of health-state monitoring, we have developed a wearable ECG based on HBC technology for monitoring driver's health-state in a car. The ECG signals are acquired by two signal electrodes and then modulated by a high-speed IR-PPM scheme in a wide frequency band from 10 to $60 \mathrm{MHz}$. The modulated ECG signals are transmitted by the two sensing electrodes through the human body. The common use of the two electrodes acting for both signal sensing and transmitting has largely simplified the wearable ECG structure. Moreover, we have also employed a moving average algorithm in the receiver side for removing the noises in the received ECG signals due to the car's movement. As a result, we have confirmed a real time ECG transmission of driver in the moving car with a reasonable accuracy.

The future subject is to further improve the filtering algorithm for removing various possible noises in driving.

\section{ACKNOWLEDGMENTS}

This study was supported in part by JSPS KAKENHI Grant Number $15 \mathrm{H} 04006$.

\section{REFERENCES}

[1] J. Wang and Q. Wang, Body Area Communications, Wiley-IEEE, 2013.

[2] IEEE Std 802.15.6-2012: IEEE Standard for local and metropolitan area networks - Part 15.6: Wireless Body Area Networks, 2012.

[3] T. G. Zimmerman, "Personal area networks: Near-field intrabody communications," IBM Syst. J., Vol. 35, No. 3\&4, pp. 609-617, 1996.

[4] M. Shinagawa, M. Fukumoto, K. Ochiai, and H. Kyuragi, "A nearfield-sensing transceiver for intrabody communication based on the electrooptic effect," IEEE Trans. Instrum. Meas., vol. 53, no. 12, pp.1533-1538, Dec. 2004.

[5] J. Wang, Y. Nishikawa and T. Shibata, "Analysis of on-body transmission mechanism and characteristic based on an electromagnetic field approach," IEEE Trans. Microw. Theory Tech., vol. 57, no. 10, pp. 2464-2470, Oct. 2009.

[6] J. Wang, T. Fujiwara and D. Anzai, "Development of impulse radio HBC transceiver for vital signal monitoring of drivers", Proc. 8th Inter. Conf. on Body Area Networks, Boston, USA, Sept. 30 - Oct. 2, 2013. 